\title{
Age related differences in acute coronary syndrome: An observation at a central hospital in Vietnam
}

\author{
Dinh The Anh ${ }^{1}$, Huynh Van Minh ${ }^{2}$, Ho Anh Binh ${ }^{3^{*}}$, Tran Quang Bao ${ }^{3}$, Nguyen Thi \\ Thai $\mathrm{Hai}^{4}$, Le Xuan Nam ${ }^{5}$, Tran Duc Anh ${ }^{6}$ \\ ${ }^{1}$ Cardiac Surgical Intensive Department, Cho Ray Hospital, Vietnam; \\ 2Department of Internal Medicine, Hue University of Medicine and Pharmacy, Hue University, Vietnam; \\ ${ }^{3}$ Department of Emergency and Interventional Cardiology, Hue Central Hospital, Vietnam; \\ ${ }^{4}$ Department of Internal Cardiology, Hue Central Hospital, Vietnam; \\ ${ }^{5}$ Department of Dermatology, Hue Central Hospital, Vietnam; \\ ${ }^{6}$ Stroke center, Hue Central Hospital, Vietnam
}

Address for Correspondence: Dr. Ho Anh Binh, MD, PhD, Department of Emergency and Interventional Cardiology, Hue Central Hospital, 16 Le Loi street, Hue city, Vietnam. E-mail: drhoanhbinh@gmail.com

\begin{tabular}{|l|}
\hline Access this article online \\
\hline $\begin{array}{l}\text { Website: } \\
\text { www.intern-med.com }\end{array}$ \\
\hline DOI: \\
10.2478/jtim-2021-0012 \\
\hline Quick Response Code: \\
\hline \\
\hline \\
\hline
\end{tabular}

\section{ABSTRACT}

Background and Objectives: There is little data regarding the characteristics of young Vietnamese patients (<40 years old) who get acute coronary syndrome (ACS). This study aimed to compare the risk factors, clinical-subclinical characteristics, coronary lesions, and mortality prediction (based on the GRACE and TIMI scores) of young ACS patients with their older counterparts. Methods: A cross-sectional descriptive study was conducted amongst 69 patients with ACS at the Cardiovascular Center of Hue Central Hospital from May 2017 to December 2018. These patients were divided into two groups: 33 patients were $<40$ years old (group 1), and 36 patients were $\geq 40$ years old (group 2). Demographic data, risk factors profile, clinical-subclinical characteristics, coronary lesions, and mortality prediction were compared between the two groups. Results: Compared with group 2, group 1 had a higher proportion of severe angina (the prevalence of angina graded III-IV by CCS classification was $69.7 \%$ in group 1 versus $36.1 \%$ in group 2, $P=0.0108$ ) and lower systolic pressure (median was 120 $\mathrm{mmHg}$ in group 1 versus $135 \mathrm{mmHg}$ in group $2, P=0.006$ ). The prevalence of unstable angina and STEMI was higher in group 1 (51.5\% and $36.4 \%$ in group 1 versus $30.6 \%$ and $11.1 \%$ in group 2, respectively), while NSTEMI was higher in group 2 (58.3\% in group 2 versus $12.1 \%$ in group $1, P=0.0002$ ). The prevalence of single-vessel CAD, normal coronary angiography (CAG), nonobstructive CAD in group 1 was also higher and multi-vessel CAD was lower than group $2(45.5 \%, 33.3 \%, 12.1 \%$ and $9.1 \%$ in group 1 versus $33.3 \%, 2.8 \%, 2.8 \%$ and $61.2 \%$ in group 2, respectively). The Gensini, GRACE, and TIMI scores were lower in group 1 (median was 5; medium was 78.55 and median was 2 in group 1 versus 37.5, 130.22 and 3 in group 2, respectively). Smoking was a risk factor for obstructive CAD in group $1(\mathrm{OR}=7.12,95 \% \mathrm{Cl}$ : $1.25-40.63, P<0.05)$. Conclusion: Young patients with ACS tended to be males, smokers, and with positive familial history; the grade of angina was more severe, and systolic pressure was lower; the prevalence of unstable angina and STEMI was higher. Smoking was a risk factor for obstructive CAD in young patients.

Key words: Acute coronary syndrome, 40 years old, Vietnam

\section{INTRODUCTION}

In recent years, there has been an increase in acute coronary syndrome (ACS) cases in young people. The cardiovascular risks of these patients are usually different. Data on
ACS prevalence, risk factors, and clinical outcomes for young patients is limited. Young patients represent $0.4 \%-19 \%$ of all ACS cases, depending on the age cut-off based on the available evidence. ${ }^{[1]}$ According to the Global Burden of Disease Study 
2010, the average age of death from myocardial ischemia in men in low-income countries was ten years younger than in the high-income countries. This might be due to earlier onset ACS and myocardial ischemia as well as shorter survival for patients in lower-middle-income countries. ${ }^{[2]}$

ACS in young patients represents a serious health-economic problem that directly affects the main workforce of society. So far, we have only found very few similar studies in Vietnam, so we conducted this research to investigate some risk factors, clinical and subclinical characteristics, coronary lesions and prognosis of death based on the GRACE, TIMI scale of ACS in patients under 40 years of age and compared these with patients over 40 years old.

\section{MATERIALS AND METHODS}

A cross-sectional descriptive study was conducted in 69 patients who were diagnosed and treated for ACS at the Cardiovascular Center of Hue Central Hospital from May 2017 to December 2018. These patients were divided into two groups: 33 patients were $<40$ years old (group 1 ), and 36 patients were $\geq 40$ years old (group 2). The institutional review board of Hue Central Hospital approved this prospective study. Written informed consent of patients was obtained.

Diagnostic criteria: ACS was diagnosed by the 4th Universal Definition of Myocardial Infarction (MI) Consensus Document (2018), ${ }^{[3]}$ in which unstable angina (UA) was diagnosed according to the standards of the European Society of Cardiology (ESC) 2019. Cardiovascular risk factors such as hypertension, diabetes, and dyslipidemia were diagnosed according to the recommendations of the ESC 2018, ${ }^{[4]}$ the American Diabetes Association (ADA) 2019, ${ }^{[5]}$ the National Cholesterol Education Program: Adult Treatment Panel III (NCEP: ATPIII) $2001^{[6]}$ or a history of treatment or previous diagnosis. A family history of coronary artery disease (CAD) was defined when close relatives of patients had CAD before the age of 55 . Overweight/ obesity was defined as BMI $\geq 23 \mathrm{~kg} / \mathrm{m}^{2}$. Other variables included a history of smoking, angina, angina level (according to Canadian Cardiovascular Society (CCS) grading scale ${ }^{[7]}$ ), other angina symptoms (sweating, shortness of breath, palpitations, vomiting, nausea, or fatigue), Killip classification, systolic blood pressure (SBP), diastolic blood pressure (DBP), and heart rate. The left ventricular ejection fraction (LVEF) was measured by $2 \mathrm{D}$ ultrasound using the Simpson method to obtain a 55\% cut-off to determine if there was an EF reduction as recommended by the American Heart Association. The severity of coronary artery stenosis was calculated based on Quantitative Analysis or visual estimation software by cardiologists who had different experiences and had the same results, from which to calculate the Gensini score. Single-vessel CAD was referred to the presence of $\mathrm{a} \geq 50 \%$ stenosis of one of the three main coronary arteries: right coronary artery (RCA), left circumflex artery (LCx), and left anterior descending (LAD) while the two remaining arteries had 1\%-49\% stenosis in diameter (defined as a non-obstructive CAD) or $0 \%$ (defined as a normal coronary angiography); $a \geq$ $50 \%$ stenosis of Left main coronary artery (LM) was seen as two-vessel CAD (equivalent to $\geq 50 \%$ stenosis of both LAD and LCx). The GRACE and TIMI scores were calculated using software or spreadsheets using the available template.

All the data were analyzed using SPSS ver. 22. It was expressed as percentages (\%) for classification variables, average \pm standard deviations, or medians $(25 \%-75 \%$ quartiles) for quantitative variables. Use T-test or MannWhitney to compare two averages, Chi-square or Fisher's exact test to compare two ratios.

\section{RESULTS}

The basic characteristics of the patients are presented in Table 1. In this study, men were more predominant in the younger group $(84.8 \%$ compared to $55.6 \% ; P=0.0177)$. The prevalence of hypertension and diabetes among younger patients was lower than the older ones (30.3\% compared to $69.4 \%$, and $3 \%$ compared to $22.2 \%$, respectively), but the family history of CAD and smoking prevalence in the younger group was higher $(18.2 \%$ compared to $2.8 \%$; $P=0.0462$, and $57.6 \%$ compared to $33.3 \%$; respectively, $P=$ 0.0443). There was no significant difference in overweight/ obesity and dyslipidemia between the two groups.

Table 2 shows the clinical characteristics of the two age groups. Compared to the older patients, younger patients had worse angina (angina class III-IV accounted for $69.7 \%$ compared to $36.1 \% ; P=0.0108$ ) and lower systolic blood pressure $(P<0.05)$, but there was no significant difference in the incidence of angina or other symptoms, Killip classification, diastolic blood pressure and heart rate between the 2 groups. The proportion of patients $<40$ years old who were admitted to the hospital for unstable angina was the highest (51.5\%), followed by ST-elevation myocardial infarction (STEMI) (36.4\%) and the age group $\geq 40$ years admitted to hospital for non-ST-elevation myocardial infarction (NSTEMI) was the highest (58.3\%), followed by unstable angina (30.6\%).

The subclinical characteristics of the two age groups are shown in Table 3. The serum glucose concentration was significantly lower, and the serum creatinine concentration was significantly higher in the younger group compared to 
Anh et al: : Age related differences in acute coronary syndrome: An observation at a central hospital in Vietnam

\begin{tabular}{llll}
\hline \multicolumn{4}{l}{ Table 1: The distribution of cardiovascular risk factors between two groups } \\
\hline \multicolumn{1}{l}{ Cardiovascular risk factors } & $<\mathbf{4 0}$ years old $(\boldsymbol{n}=\mathbf{3 3})$ & $\mathbf{2} \mathbf{4 0}$ years old $(\boldsymbol{n}=\mathbf{3 6})$ & $\boldsymbol{P}$-value \\
\hline Male & $28(84.8 \%)$ & $20(55.6 \%)$ & 0.0177 \\
Overweight/Obese & $17(51.5 \%)$ & $11(30.6 \%)$ & 0.1284 \\
Smoking & $19(57.6 \%)$ & $12(33.3 \%)$ & 0.0443 \\
Hypertension & $10(30.3 \%)$ & $25(69.4 \%)$ & 0.0027 \\
Diabetes & $1(3.0 \%)$ & $8(22.2 \%)$ & 0.0445 \\
Dyslipidemia & $27(81.8 \%)$ & $33(91.7 \%)$ & 0.3886 \\
Family history of CAD & $6(18.2 \%)$ & $1(2.8 \%)$ & 0.0462 \\
\hline
\end{tabular}

\begin{tabular}{llll}
\hline Table 2: Clinical characteristics & & \\
\hline Clinical characteristics & $<\mathbf{4 0}$ years old $(\boldsymbol{n}=\mathbf{3 3})$ & $\mathbf{2} \mathbf{4 0}$ years old $(\boldsymbol{n}=\mathbf{3 6})$ & $\boldsymbol{P}$-value \\
\hline Angina & $32(97.0)$ & $33(91.7)$ & 0.6697 \\
Non-angina and angina (class I-II) & $10(30.3)$ & $23(63.9)$ & 0.0108 \\
Angina (class III-IV) & $23(69.7)$ & $13(36.1)$ & 0.0108 \\
Other symptoms (without angina) & $18(54.5)$ & $19(52.8)$ & 0.9202 \\
STEMI & $12(36.4)$ & $4(11.1)$ & 0.0277 \\
NSTEMI & $4(12.1)$ & $21(58.3)$ & 0.0002 \\
Unstable angina & $17(51.5)$ & $11(30.6)$ & 0.1284 \\
Killip $\geq I I$ & $1(3.0)$ & $5(13.9)$ & 0.2388 \\
Systolic blood pressure $(\mathrm{mmHg})$ & $120(110-135)$ & $135(120-140)$ & 0.006 \\
Diastolic blood pressure $(\mathrm{mmHg})^{*}$ & $70(70-80)$ & $80(72.5-80)$ & 0.1712 \\
Heart rate (beats/min) & $75(68-90)$ & $80(71.3-84.8)$ & 0.4916 \\
\hline
\end{tabular}

(*) Medians (25\%-75\% quartiles)

\begin{tabular}{llll}
\hline \multicolumn{2}{l}{ Table 3: Subclinical characteristics } \\
\hline $\begin{array}{l}\text { Subclinical characteristics } \\
\text { Medians (25\%-75\% quartiles) }\end{array}$ & $<\mathbf{4 0}$ years old $(\boldsymbol{n}=\mathbf{3 3})$ & $\mathbf{2} \mathbf{4 0}$ years old $(\boldsymbol{n}=\mathbf{3 6})$ & $\boldsymbol{P}$-value \\
\hline Glucose (mmol/L) & $5.3(4.66-6.6)$ & $6.44(5.51-9.23)$ & 0.001 \\
Total cholesterol (mmol/L) & $4.83(4.07-5.44)$ & $4.38(3.59-5.42)$ & 0.256 \\
Triglyceride (mmol/L) & $1.68(1.17-2.79)$ & $2(1.21-3.01)$ & 0.657 \\
HDL-cholesterol (mmol/L) & $1.02(0.95-1.13)$ & $2(1.21-3.01)$ & 0.862 \\
LDL-cholesterol (mmol/L) & $2.9(2.47-3.71)$ & $2.95(1.98-3.58)$ & 0.759 \\
CK (U/L) & $104(72.5-347)$ & $2.96(1.41-5.49)$ & 0.552 \\
CK-MB (ng/mL) & $1.93(1.13-4.98)$ & $2.96(1.41-5.49)$ & 0.223 \\
hs Troponin T (ng/mL) & $0.01(0.007-0.665)$ & $0.024(0.011-0.104)$ & 0.234 \\
Creatinine ( $\mu \mathrm{mol} / \mathrm{L})$ & $80(68-94.5)$ & $72.5(60.5-83.75)$ & 0.031 \\
\hline
\end{tabular}

the older group. Levels of total cholesterol, triglycerides, LDL and HDL cholesterol, CK, CK-MB, hs Troponin T were similar between the two groups.

Compared to the age group $\geq 40$ years, the proportion of patients with ST-segment elevation was significantly higher in the $<40$ years group, in contrast to the significantly lower proportion of ST-segment depression or isoelectricity. There was no significant difference in the incidence of arrhythmia, as well as the reduction in EF and mean EF between the 2 age groups (Table 4).

Table 5 shows the characteristics of coronary artery lesions. Compared to the patients aged $\geq 40$ years, the incidence of LAD, LCx, and RCA lesions were lower in the $<40$-year-old group. The rate of single-vessel
CAD, normal coronary angiography (CAG), coronary artery stenosis was insignificant in the patients $<40$ years of age and the rate of muti-vessel CAD was significantly lower than the age group $\geq 40$ years. There was no difference in the prevalence of LM lesions and myocardial bridging between the two age groups. The patients $<40$ years of age had significantly lower Gensini, GRACE, and TIMI scores than the $\geq 40$-year-old group (Table 6).

\section{DISCUSSION}

Our research showed that ACS among young people was more common in men. The proportion of smoking and family history of CAD among the younger patients was higher than the older ones, but the prevalence of 
Anh et al: : Age related differences in acute coronary syndrome: An observation at a central hospital in Vietnam

\begin{tabular}{|c|c|c|c|}
\hline Characteristics of ECG and LVEF & $<40$ years $(n=33)$ & $\geq 40$ years $(n=36)$ & $P$-value \\
\hline Normal sinus rhythm & $25(75.8 \%)$ & $32(88.9 \%)$ & 0.2641 \\
\hline Arrhythmia & $8(24.2 \%)$ & $4(11.1 \%)$ & 0.2641 \\
\hline ST segment elevation & $12(36.4 \%)$ & $4(11.1 \%)$ & 0.0277 \\
\hline ST segment depression & $8(24.2 \%)$ & $10(27.8 \%)$ & 0.9476 \\
\hline ST-segment isoelectric & $13(39.4 \%)$ & $22(61.1 \%)$ & 0.1188 \\
\hline Reduced EF & $11(33.3 \%)$ & $16(44.4 \%)$ & 0.4858 \\
\hline Mean EF \pm standard deviation & $60.02 \pm 9.97$ & $56.22 \pm 11.68$ & 0.1526 \\
\hline
\end{tabular}

\begin{tabular}{llll}
\hline \multicolumn{1}{l}{ Table 5: Characteristics of coronary artery lesions } \\
\hline Coronary artery lesions & $<\mathbf{4 0}$ years $(\boldsymbol{n}=\mathbf{3 3})$ & $\mathbf{2} \mathbf{4 0}$ years $(\boldsymbol{n}=\mathbf{3 6})$ & $\boldsymbol{P}$-value \\
\hline LM lesions & $1(3.0)$ & $7(19.4)$ & 0.0798 \\
LAD lesions & $17(51.5)$ & $32(88.9)$ & 0.0016 \\
LCx lesions & $3(9.1)$ & $17(47.2)$ & 0.0013 \\
RCA lesions & $6(18.2)$ & $22(61.1)$ & 0.0007 \\
Normal CAG & $11(33.3)$ & $1(2.8)$ & 0.0025 \\
Nonobstructive CAD & $4(12.1)$ & $1(2.8)$ & 0.3060 \\
Single-vessel CAD & $15(45.5)$ & $12(33.3)$ & 0.4293 \\
Two-vessels CAD & $1(3.0)$ & $11(30.6)$ & 0.0069 \\
Three-vessels CAD & $2(6.1)$ & $11(30.6)$ & 0.0220 \\
Myocardial bridging & $7(21.2)$ & $2(5.6)$ & 0.1180 \\
\hline
\end{tabular}

\begin{tabular}{llll}
\hline Table 6: Gensini, GRACE and TIMI score & & & \\
\hline Gensini, GRACE, and TIMI score & $<\mathbf{4 0}$ years $(\boldsymbol{n}=\mathbf{3 3})$ & $\mathbf{2 4 0}$ years $(\boldsymbol{n}=\mathbf{3 6})$ & $\boldsymbol{P}$-value \\
\hline Gensini score (median, range) & $5(0-32)$ & $37.5(13.25-75.5)$ & 0.001 \\
GRACE score (Mean \pm standard deviation) & $78.55 \pm 26.23$ & $130.22 \pm 31.6$ & 0.001 \\
TIMI score (median, range) & $2(1-3)$ & $3(2-4)$ & 0.003 \\
\hline
\end{tabular}

hypertension and diabetes in this group was less common; this was consistent with the majority of previous domestic and foreign studies. ${ }^{\left[{ }^{8-11]}\right.}$ Smoking and dyslipidemia had been reported as the most important cardiovascular risk factors for young patients with myocardial infarction. ${ }^{[1]}$ Our research also showed the same thing in young ACS patients. In addition, the predominance of a family history of $\mathrm{CAD}$ in young ACS patients had also been recorded through many studies. ${ }^{[11-13]}$ This highlighted the role of genetic factors in the cause of ACS among young individuals.

In our study, the distribution of ACS types was markedly different when the rates of unstable angina and STEMI were significantly higher in the younger patients compared to the older ones. According to Klein et al. (1987), most young CAD patients had no previous history of angina until being hospitalized for acute myocardial infarction. ${ }^{[14]}$ Data on STEMI prevalence in young ACS patients varied widely in different studies-ranging from $6 \%-73 \%$, but all of them had in common that the proportion of STEMI in young patients was higher when compared with the group of elderly patients. ${ }^{[12,13,15,16]}$
In this study, younger patients had worse angina and lowered systolic blood pressure. In the literature, there is little available comparative data related to angina in young ACS patients. Most previous studies reporting data on angina have just focused on young patients with MI or ACS in general. ${ }^{[11,17]}$

Younger ACS patients had lower plasma glucose concentrations and higher plasma creatinine concentrations than older individuals. This can be explained by the fact that younger people are less likely to suffer from diabetes, and they often have a larger lean mass as well as a higher meat diet than older people. However, comparative data relating to subclinical characteristics in young ACS patients is still limited; some previous studies have shown different results, possibly due to differences in sample selection and anthropometry characteristics of research subjects. ${ }^{[16,18,19]}$

Younger patients with ACS had a higher incidence of normal CAG and non-obstructive CAD than the older ones. The pathogenesis of ACS in young patients without obstructive CAD may be related to coronary artery spasm, spontaneous coronary artery dissection, endothelial 
dysfunction, myocardial bridging, or non-coronary causes (e.g., anemia, respiratory failure, hypovolemic shock, tachycardia/slow arrhythmia). Similar to other studies, the majority of single-vessel CAD was in younger patients, while most multi-vessel CAD was more common in older patients. ${ }^{[17,20]}$

Compared to the older patients, the younger ACS ones had lower coronary artery lesions on the Gensini score and a lower prognosis of death on the GRACE, TIMI scores. In other words, the younger patients have a better prognosis than the older ones. This has also been noted in previous studies. ${ }^{[21,22]}$

\section{CONCLUSION}

ACS patients in young adults had a higher incidence in males accompanied with smoking and family medical history of CAD; the grade of angina was more severe and systolic blood pressure was lower; the prevalence of Unstable angina and STEMI was also higher compared to the elderly patients. Elderly patients had higher blood glucose levels and lower creatinine levels; the rate of CAD with multi-vessel disease was higher in the young patients. Additionally, in the young patients, the Gensini, GRACE, and TIMI scores were lower, and smoking was the risk factor for obstructive CAD.

Further efforts are needed to prevent smoking among adolescents to avoid and overcome smoking addiction. Clinicians should carefully assess the suspicion of ACS in young people to avoid missing the diagnosis of ACS.

\section{Author's contribution}

All the authors participated in the study design, data collection, and literature search. Data was analyzed by DTA. $\mathrm{HAB}$ wrote the paper. All the authors read and approved the final manuscript.

\section{Funding}

No funding was received

\section{Conflicts of Interest}

The authors declare no conflict of interest

\section{Acknowledgments}

None

\section{REFERENCES}

1. Puricel S, Lehner C, Oberhänsli M, Rutz T, Togni M, Stadelmann M, et al. Acute coronary syndrome in patients younger than 30 years-aetiologies, baseline characteristics and long-term clinical outcome. Swiss medical weekly 2013; 143: w13816.

2. Vedanthan R, Seligman B and Fuster V. Global Perspective on Acute Coronary Syndrome: A Burden on the Young and Poor. Circulation research 2014; 114: 1959-75.

3. Thygesen K, Alpert JS, Jaffe AS, Chaitman BR, Bax JJ, Morrow DA, et al. Fourth Universal Definition of Myocardial Infarction (2018). Circulation 2018; 138: e618-e51.

4. Williams B, Mancia G, Spiering W, Agabiti Rosei E, Azizi M, Burnier M, et al. 2018 ESC/ESH Guidelines for the management of arterial hypertension: The Task Force for the management of arterial hypertension of the European Society of Cardiology and the European Society of Hypertension: The Task Force for the management of arterial hypertension of the European Society of Cardiology and the European Society of Hypertension. Journal of hypertension 2018; 36: 1953-2041.

5. American Diabetes A. 10. Cardiovascular Disease and Risk Management: Standards of Medical Care in Diabetes-2019. Diabetes care 2019; 42: S103-S23.

6. Lipsy RJ. The National Cholesterol Education Program Adult Treatment Panel III guidelines. Journal of managed care pharmacy : JMCP 2003; 9: $2-5$.

7. Smith ER. The angina grading system of the Canadian Cardiovascular Society. The Canadian journal of cardiology 2002; 18: 439, 42.

8. Chen TS-C, Incani A, Butler TC, Poon K, Fu J, Savage M, et al. The Demographic Profile of Young Patients ( $<45$ years-old) with Acute Coronary Syndromes in Queensland. Heart, Lung and Circulation 2014; 23: 49-55.

9. Cong ND and Dung HT. The risk factors of acute coronary syndrome in patients over 65 years old at Thong Nhat Hospital of Ho Chi Minh City, Vietnam. Journal of atherosclerosis and thrombosis 2014; 21: S36-S41.

10. Obaya M, Yehia M, Hamed L and Fattah AA. Comparative study between elderly and younger patients with acute coronary syndrome. The Egyptian Journal of Critical Care Medicine 2015; 3: 69-75.

11. Schoenenberger AW, Radovanovic D, Stauffer J-C, Windecker S, Urban P, Niedermaier G, et al. Acute coronary syndromes in young patients: Presentation, treatment and outcome. International Journal of Cardiology 2011; 148: 300-4.

12. Panduranga P, Sulaiman K, Al-Zakwani I and Abdelrahman S. Acute coronary syndrome in young adults from oman: results from the gulf registry of acute coronary events. Heart views: the official journal of the Gulf Heart Association 2010; 11: 93.

13. Ricci B, Cenko E, Vasiljevic Z, Stankovic G, Kedev S, Kalpak O, et al. Acute coronary syndrome: the risk to young women. Journal of the American Heart Association 2017; 6: e007519.

14. Klein LW, Agarwal JB, Herlich MB, Leary TM and Helfant RH. Prognosis of symptomatic coronary artery disease in young adults aged 40 years or less. The American journal of cardiology 1987; 60: 1269-72.

15. Avezum A, Makdisse M, Spencer F, Gore JM, Fox KA, Montalescot G, et al. Impact of age on management and outcome of acute coronary syndrome: observations from the Global Registry of Acute Coronary Events (GRACE). American heart journal 2005; 149: 67-73.

16. Ma Q, Wang J, Jin J, Gao M, Liu F, Zhou S, et al. Clinical characteristics and prognosis of acute coronary syndrome in young women and men: A systematic review and meta-analysis of prospective studies. International Journal of Cardiology 2017; 228: 837-43.

17. Zimmerman FH, Cameron A, Fisher LD and Grace N. Myocardial infarction in young adults: angiographic characterization, risk factors and prognosis (Coronary Artery Surgery Study Registry). Journal of the American College of Cardiology 1995; 26: 654-61. 
18. Chye NCY, Khir RN, Wen LC, Ismail JR, Rahman EA, Aiza H, et al. Young acute coronary syndrome outcomes in heterogenous Asians. Journal of Cardiovascular Medicine and Therapeutics 2018; 2: 1-5.

19. Ge J, Li J, Yu H and Hou B. Hypertension Is an Independent Predictor of Multivessel Coronary Artery Disease in Young Adults with Acute Coronary Syndrome. International journal of hypertension 2018; 2018.

20. Maurya RK, Satish L, Sanghvi S and Goutam K. Coronary angiographic profile characteristics in young patients with acute coronary syndrome and comparison with older patients with acute coronary syndrome. International Journal of Research in Medical Sciences 2016; 4: 1415-8.

21. Mahjoob MP, Sadeghi S, Khanaman HF, Naderian M and Khaheshi I. Comparison of coronary risk factors and angiographic findings in younger and older patients with significant coronary artery disease. Romanian Journal of Internal Medicine 2018; 56: 90-5.
22. Tini G, Proietti G, Casenghi M, Colopi M, Bontempi K, Autore C, et al. Long-Term Outcome of Acute Coronary Syndromes in Young Patients. High Blood Pressure \& Cardiovascular Prevention 2017; 24: 77-84.

How to cite this article: Anh DT, Minh HV, Binh HA, Bao TQ, Hai NTT, Nam LX, et al. Age related differences in acute coronary syndrome: An observation at a central hospital in Vietnam. J Transl Intern Med 2021; 9: $32-7$ 